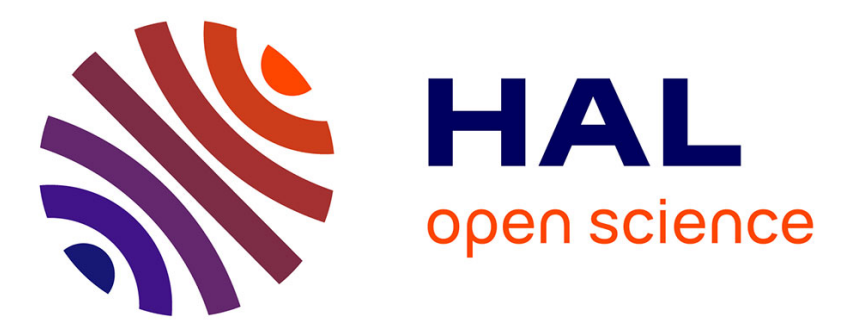

\title{
Measuring and Mitigating Web Performance Bottlenecks in Broadband Access Networks
}

Srikanth Sundaresan, Nick Feamster, Renata Teixeira, Nazanin Magharei

\section{To cite this version:}

Srikanth Sundaresan, Nick Feamster, Renata Teixeira, Nazanin Magharei. Measuring and Mitigating Web Performance Bottlenecks in Broadband Access Networks. ACM Internet Measurement Conference, Oct 2013, Barcelone, Spain. pp.213-226, 10.1145/2504730.2504741 . hal-00937167

\section{HAL Id: hal-00937167 https://hal.sorbonne-universite.fr/hal-00937167}

Submitted on 27 Jan 2014

HAL is a multi-disciplinary open access archive for the deposit and dissemination of scientific research documents, whether they are published or not. The documents may come from teaching and research institutions in France or abroad, or from public or private research centers.
L'archive ouverte pluridisciplinaire HAL, est destinée au dépôt et à la diffusion de documents scientifiques de niveau recherche, publiés ou non, émanant des établissements d'enseignement et de recherche français ou étrangers, des laboratoires publics ou privés. 


\section{Measuring and Mitigating Web Performance Bottlenecks in Broadband Access Networks}

\author{
Srikanth Sundaresan \\ Georgia Tech \\ srikanth@gatech.edu \\ Renata Teixeira \\ CNRS \& UPMC \\ renata.teixeira@lip6.fr
}

\author{
Nick Feamster \\ Georgia Tech \\ feamster@cc.gatech.edu \\ Nazanin Magharei \\ Cisco Systems \\ n.magharei@gmail.com
}

\begin{abstract}
We measure Web performance bottlenecks in home broadband access networks and evaluate ways to mitigate these bottlenecks with caching within home networks. We first measure Web performance bottlenecks to nine popular Web sites from more than 5,000 broadband access networks and demonstrate that when the downstream throughput of the access link exceeds about $16 \mathrm{Mbits} / \mathrm{s}$, latency is the main bottleneck for Web page load time. Next, we use a routerbased Web measurement tool, Mirage, to deconstruct Web page load time into its constituent components (DNS lookup, TCP connection setup, object download) and show that simple latency optimizations can yield significant improvements in overall page load times. We then present a case for placing a cache in the home network and deploy three common optimizations: DNS caching, TCP connection caching, and content caching. We show that caching only DNS and TCP connections yields significant improvements in page load time, even when the user's browser is already performing similar independent optimizations. Finally, we use traces from real homes to demonstrate how prefetching DNS and TCP connections for popular sites in a home-router cache can achieve faster page load times.
\end{abstract}

\section{Categories and Subject Descriptors}

C.2.3 [Computer-Communication Networks]: Network Operations-Network Management; C.2.3 [Computer-Communication Networks]: Network Operations-Network Monitoring

\section{Keywords}

Broadband Networks; Web performance; Bottlenecks; Popularitybased prefetching; Connection caching; DNS prefetching

\section{INTRODUCTION}

Home broadband Internet access is getting faster: the OECD reports that broadband speeds are increasing by about $15-20 \% \mathrm{ev}-$ ery year. Average advertised broadband speeds are now about $16 \mathrm{Mbits} / \mathrm{s}$ in the U.S. and 37.5 Mbits/s across OECD areas [45].

Permission to make digital or hard copies of all or part of this work for personal or classroom use is granted without fee provided that copies are not made or distributed for profit or commercial advantage and that copies bear this notice and the full citation on the first page. Copyrights for components of this work owned by others than ACM must be honored. Abstracting with credit is permitted. To copy otherwise, or republish, to post on servers or to redistribute to lists, requires prior specific permission and/or a fee. Request permissions from permissions@acm.org.

IMC'13, October 23-25, 2013, Barcelona, Spain.

Copyright 2013 ACM 978-1-4503-1953-9/13/10 ...\$15.00

http://dx.doi.org/10.1145/2504730.2504741
As downstream throughput continues to increase, one might expect the Web to get faster at home, as well. Meanwhile, Internet service providers and application providers are increasingly cognizant of the importance of reducing Web page load times; even seemingly small differences in latency can introduce significant effects on usability (and revenue). The Bing search engine experiences reduced revenue of $1.2 \%$ with just a 500-millisecond delay [53], and a 400 -millisecond delay resulted in a $0.74 \%$ decrease in searches on the Google search engine [16]. Forrester research found that most users expected online shopping sites to load in two seconds or fewer [42]. Content providers struggle to mitigate any network performance bottleneck that can slow down Web page loads in access networks by even tens of milliseconds. Thus, it is crucial to understand both how network properties of access networks such as latency can introduce bottlenecks in Web page load times and the extent to which various optimizations can help further mitigate these bottlenecks.

Towards this goal, in this paper, we use measurements from a router-based Web performance measurement tool, Mirage, to analyze Web page load times to nine popular Web sites. This tool has been deployed in over 5,000 home networks as part of the FCC/SamKnows deployment in the US. We also deploy the tool in our own smaller deployment, BISmark. We examine how access network latency and throughput can introduce performance bottlenecks and evaluate how to mitigate these bottlenecks by deploying various caching optimizations on the router in the home network. Next, we demonstrate that caching on a home router can improve page load time, even if the router does not cache any content (i.e., even if it only caches DNS records and TCP connections), and even if the end host or Web browser is already independently performing similar optimizations. Finally, we show how prefetching DNS records and TCP connections from the home router can improve cache hit rates; we use a trace-based emulation to show that prefetching can help achieve these performance improvements in real home networks. We now describe each of these contributions in more detail.

First, we measure Web performance from 5,556 broadband access networks to nine popular Web sites and identify bottlenecks that contribute to Web page load time in these networks (Section 5). Our results suggest that latency is the main bottleneck for Web page load times for access links whose downstream throughput exceeds about $16 \mathrm{Mbits} / \mathrm{s}$. Last-mile latency is an important contributor to the end-to-end latency, and an increase in last-mile latency of just 10 milliseconds can sometimes induce delays of hundreds of milliseconds for page load times of popular sites. In the case of small objects, we find that TCP latency overhead exceeds the actual download time of the object. Our results corroborate and quantify 
anecdotal evidence from users, Internet service providers, and content providers who are increasingly finding that latency is becoming a critical performance bottleneck [30,42].

Second, we use Mirage to deconstruct page load time into its constituent components (i.e., DNS lookup, TCP connection setup, content download) and show that even small improvements in latency can yield significant improvements in overall page load times (Section 6). To our knowledge, this paper presents the first study to compare the relative benefits of content caching, DNS caching, and TCP connection caching from within home networks. As part of this analysis, we explore how the page load time that Mirage measures relates to Web page load time measured by other tools (e.g., Phantomjs, Web browsers). We find that latency is a significant contributor to all factors that affect page load time. These results-in conjunction with previous studies that have observed that characteristics of the access network can introduce significant latency [55] - present the case for home caching, the process of caching DNS lookups, TCP connections, and content from the home router.

Third, we deploy an OpenWrt module that performs various caching optimizations in home routers on the BISmark testbed and show that such a cache can yield improvements in page load time, even if it does not cache content, and even if the browser is already performing similar optimizations (Section 7). As expected, content caching offers the most significant reductions in page load time and can reduce page load times by up to $53 \%$ in some cases. Yet, simply caching TCP connections and DNS records at the home router can reduce mean page load times by $20 \%$ and $7 \%$, respectively, even if the ISP and browser are also independently performing their own caching optimizations.

Finally, to improve cache hit rates in real home networks, we introduce popularity-based prefetching, which prefetches DNS records and TCP connections for Web sites that are commonly accessed from a home network. Using Web traces from twelve home networks, we find that prefetching DNS records and TCP connections for only the twenty most popular sites significantly increases DNS and connection cache hit rates and therefore could offer significant improvements in page load times.

The Web performance measurements from the SamKnows deployment are available on the FCC Web site [?]. We have published both the Web performance measurements from the BISmark experiments [15] and the OpenWrt module that performs popularitybased prefetching [50].

\section{BACKGROUND}

Factors that affect page load time. Web downloads begin by downloading the home page of the requested Web page. The home page object typically contains references to other objects that the browser must subsequently retrieve. Each of these objects is referenced with another URL, which the browser retrieves with additional HTTP requests. These objects are either static, in which case the URL is in the home page itself; or dynamic, in which case the URL is determined by running active scripts (e.g., Javascript) that determine which objects to download. Modern Web sites typically contain a mix of static and dynamic objects. Browsers minimize the download time of the individual objects using many optimizations such as parallel fetches, DNS caching, TCP connection reuse, and optimizations for processing scripts and rendering. Different browsers implement different sets of optimizations.

Because the time for the browser to render a page depends on the choice of browser and machine configuration, we instead define page load time as the time from the initial request to the time when all objects for the page have been retrieved. The retrieval time for each object has the following components: (1) the DNS lookup time for the domain referenced in the object URL; (2) the TCP connection time, which is the time to complete the TCP threeway handshake to the server; (3) the server response time, which is the time it takes for the server to start sending the object once it has been requested; and (4) the object download time, which is the time to download the object itself over TCP. Some of these factors, such as the DNS lookup and TCP connection times, are bound by latency; others, such as the object download time, are bound by both latency and throughput.

Optimizations to improve page load time. A number of optimizations have been proposed and implemented in the quest to minimize page load time. Server-side optimizations include HTTP replacements [?, 54] and TCP modifications [13, 14, 21, 22, 28]. Recent proposals suggest using a larger initial congestion window sizes on servers for TCP connection, so that small objects can be transferred with significantly fewer round trips [28]. Al-Fares et al. studied the effects of server-side optimizations, such as increasing TCP's initial congestion window (ICW) and enabling HTTP pipelining on Web page load times [9]. They found that increasing the ICW can reduce page load times by several hundred milliseconds in many cases. Although these server-side optimizations can improve page load times, they do not reduce certain components that contribute to page load time, including DNS lookup and the TCP connection setup time.

Many client-side optimizations from the browser have also been developed. HTTP/1.1 introduced persistent connections and pipelining. Persistent connections allow the client to retrieve multiple objects over the same TCP connection (thereby amortizing the three-way handshake and TCP congestion window ramp up cost over multiple objects). Pipelining allows the client to initiate a request for the next object as soon as it sees a reference to that object in another object (rather than waiting for the object download to complete). Nielsen et al. showed the superior performance of HTTP/1.1 over HTTP/1.0 [44]. However, most browsers do not enable pipelining by default, and some servers do not enable persistent connections. Zhou et al. propose a new protocol that minimizes connection time by having DNS resolvers set up TCP connection on the client's behalf [60]. Browsers also download multiple objects in parallel and have highly optimized engines for parsing and rendering objects.

Content caching is also a common optimization. Content Distribution Networks (CDNs) are large distributed caches that are typically deployed at the edge of ISPs to reduce the latency between the end-host and the content. Ihm et al. characterized five years of Web traffic traces from a globally distributed proxy service; they observe that Web caches typically have a $15-25 \%$ hit rate, and these rates could almost double if caches operated on 128-byte blocks [33]. Previous studies have reported object cache hit rates in the range of 35-50\%, although these cache hit rates have continued to drop over time $[4,18,32,43,59]$. Some browsers also support content prefetching [41]; Padmanabhan et al. proposed predictive content prefetching using server hints [47].

To improve cache hit ratios, Web browsers prefetch DNS records anticipating client requests; the browser parses certain downloaded pages (e.g., a search result page) for domains and resolves them before the user clicks on them [26]. To reduce the time associated with DNS lookups, browsers and intermediate DNS servers employ caching and prefetching $[23,24]$. Jung et al. studied DNS performance and the effectiveness of DNS caching [36], and saw that DNS cache hit rates can be as high as $80 \%$, even with only a few 
clients [35]. Feldmann et al. observed in Web traces from AT\&T home users that $47 \%$ of objects retrieved incur more than half of the total download time from TCP connection setup [31]. Based on this observation, the study proposes a connection cache in the ISP network to reduce connection setup time, which reduces download times by up to $40 \%$.

\section{RELATED WORK}

There is much previous work on measuring and modeling Web performance, ranging from protocol modeling to empirical analysis to designing techniques to improve performance. Many of these previous studies were performed before the growth of broadband access networks, content distribution networks, and modern browsers. This evolution suggests that it is time for a reappraisal of some past studies in the context of broadband access networks, although it is important to put our study in the context of previous work. Below, we compare past work in measuring and modeling Web performance to the study in this paper. This study is a more complete version of our own previously published work, which explored the contribution of latency to Web page load time [57] and the positive performance benefits of proactive caching and prefetching [56]

Measuring Web performance. Barford et al. analyzed the contribution of server, client, and network delays to HTTP 1.0 transaction times [12]; the authors used virtual clients on several campus networks to request files from a virtual Web server. They found that for small files, server load is the dominant factor for performance, while for large files, the network properties dominate. $\mathrm{Kr}-$ ishnamurthy and Wills analyzed the impact of variants of TCP (i.e., Tahoe, Reno) and HTTP (i.e., persistent, parallel, or pipelined) on Web performance; they also studied the effects of network latency and server load on observed performance [38]. These studies were performed more than ten years ago. During this time period, the Web has changed dramatically, in terms of the type of content is served, how content is hosted, and how browsers operate. Additionally, broadband Internet access has proliferated since the late 1990s. It is also much faster than it was when these original studies were conducted and is now a predominant mode of Internet access. To our knowledge, this study is the first to explore Web page load times from broadband access links, and the first to quantify the extent to which latency becomes a bottleneck on high-throughput access links.

More recent work has studied the performance of CDNs [34] and caching [29]. Akella et al. [5-8] studied the effects of the performance of $68 \mathrm{CDN}$ sites across 17 cities, focusing on how server multihoming can improve CDN performance for clients. "WhyHigh?" identifies cases where a certain set of clients experience higher Web page load times [39]. Butkiewicz et al. recently studied how the complexity of modern Web sites may contribute to slower page load times and found that more than $60 \%$ of the Web sites they profiled retrieve content from five non-origin sources that contribute to more than $35 \%$ of the bytes downloaded [17]. Our work complements existing literature by exposing how access networks introduce bottlenecks and assessing how well existing Web performance optimizations work when they are deployed in homes.

The online service webpagetest .org [?] analyzes the contribution of different network components to overall page load time via a "waterfall"; the analysis is similar to the decomposition that we perform in this paper, although the software we develop can run directly on home routers, unlike webpagetest. org, which runs from data centers. Keynote [37] and Compuware [25] perform endto-end Web performance measurements from a variety of vantage

\begin{tabular}{|l|l|l|}
\hline Metric & Type & Description \\
\hline Page load time & Total & $\begin{array}{l}\text { The time to look up DNS, set up TCP } \\
\text { connections and retrieve all objects. } \\
\text { The DNS lookout time for the main } \\
\text { domain of the site. } \\
\text { The time from the initiation of the } \\
\text { TCP connection to the arrival of the } \\
\text { first byte of the requested object (in- } \\
\text { cluding server processing time) } \\
\text { The time to download an object, ex- } \\
\text { cluding the DNS lookup time and } \\
\text { time to first byte. }\end{array}$ \\
\hline
\end{tabular}

Table 1: Performance metrics. For each per-object metric, Mirage measures the maximum, minimum, and average times for each object in the transaction.

points, but they do not study how individual network factors such as latency, loss, or throughput ultimately contribute to page load time.

Modeling Web performance. Previous work developed models for TCP and HTTP performance based on various network properties such as latency and packet loss $[10,11,19,20,46]$. We present an empirical study of Web performance from thousands of access networks. This empirical approach provides new insights into how access network characteristics affect each component of page load time. WebProphet [40] and WProf [58] analyze dependencies between Web page objects to predict browser-level response time and study bottlenecks in the critical path of page load times. These tools are valuable in the quest to improve page load times by exposing inefficiencies in Web site design, but they are difficult to deploy at scale.

\section{MEASURING PAGE LOAD TIME}

The notion of page load time depends on many factors, including the underlying network, the design of the Web site, and the endhost, including the browser. Every browser downloads pages using different methods (and different optimizations), and the pages themselves may be optimized for different browsers and devices. In this paper, we explore how the characteristics of broadband networks affect Web page load times. Page load time will necessarily vary depending on the above characteristics. Thus, to conduct controlled experiments of the effects of network characteristics on page load time, we use a single tool on a common platform to measure page load times as various network conditions change. We now explain the design of the active measurement tool that we use, and how the numbers it yields compares to that of a browser.

\subsection{Mirage: Home Router-Based Web Testing}

We use measurements from the FCC/SamKnows deployment of routers across the US [55]. The deployment uses a custom tool, which we call Mirage, to measure page load time. Mirage is a headless Web client designed by SamKnows for deployment on home router devices; the initial deployment of Mirage was sponsored by the Federal Communications Commission (FCC) on more than 5,000 homes across the US. We also use it in the BISmark deployment.

\subsubsection{How Mirage Works}

Mirage downloads the home page of a Web site and parses it to determine the static objects that are needed to render the page. It then performs all the DNS lookups at once before downloading the rest of the objects. The tool is based on libcurl, which can de- 


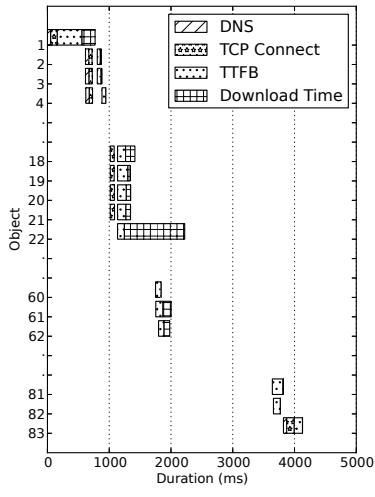

(a) Real Browser

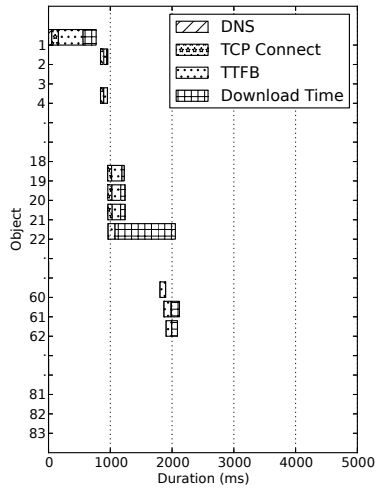

(b) Mirage
Figure 1: Comparison of a real browser (from webpagetest.org) with Mirage for www.ebay.com; some objects are omitted. The browser optimizes object retrieval differently, and also retrieves more objects. Still, the performance bottlenecks are similar for both.

compose the overall page load time into the download times for individual objects. Mirage separates the page load time into the time to perform DNS lookups, the time to first byte (which combines the TCP connection time and the server processing time), and the actual load time for each object. It uses persistent TCP connections if the server supports them and up to eight parallel TCP connections to download objects. Because Mirage uses many of the basic network optimizations that a browser uses including persistent TCP connections and parallel TCP connections, it approximates the that a real browser might see, even though it does not emulate any particular browser. Table 1 shows the performance metrics for each download and how the performance measurements from Mirage compare to those from webpagetest. org.

Mirage is ideal for our goal of studying the effect of the characteristics of broadband access networks on Web performance for a number of reasons. First, because Mirage can be deployed directly on home routers, it provides measurements from a single platform that is directly connected to the access link, thereby normalizing some of the effects that might otherwise affect page load times (e.g., the use of different browsers, home network effects). Second, Mirage is already in use in the large FCC/SamKnows deployment, which provides longitudinal measurements of the same set of diverse access links. Because Mirage breaks down each object into its constituent parts, it exposes important performance bottlenecks in access networks. Finally, Mirage is freely available and portable; we have used Mirage in our own BISmark deployment.

\subsubsection{Validation}

Comparing Mirage to real browser behavior. Mirage's behavior differs from a browser in several ways. We explain these differences using the example download in Figure 1. This figure shows the partial breakdown of the page load time for Ebay both using a real browser through webpagetest.org and Mirage. First, Mirage waits to download the home page before processing it and downloading subsequent objects; in contrast, many modern browsers start downloading objects as they process the home page. This difference is visible in objects 2-4 in Figure 1, where the real

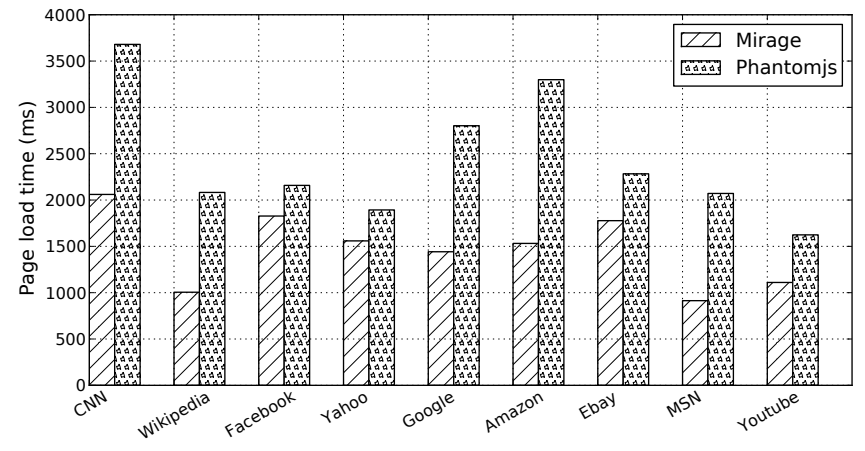

Figure 2: Comparison of Mirage to Phantomjs. We see that Mirage underestimates the page load times: real load times are higher than what we measure.

browser initiates the download before object 1 is complete. Mirage also performs DNS queries for all the unique domains after parsing the home page before downloading any of the remaining objects; it adds the maximum DNS time to the total time to download the objects. Although Mirage performs the DNS queries before downloading any objects, the effect on total page load time is not significant. The time to retrieve each individual object and the time spent on each component of the object retrieval is nearly identical for the case of the real browser and Mirage.

Mirage also downloads a slightly different, smaller set of objects than a real browser. Modern Web sites, especially content-heavy ones, employ active scripts. These scripts result in additional processing latency and also frequently result in the browser downloading more objects. Mirage only processes static objects, so it downloads a subset of the objects that are downloaded by a real browser, usually resulting in a smaller page load time than a normal browser would see. For example, in Figure 1, the browser downloads 83 objects, while Mirage downloads 62 objects.

Validation with Phantomjs. To understand how the differences between Mirage and a real browser affect overall page load time, we compare Mirage's measurements to those of a real browser environment. For this comparison, we use Phantomjs [48], a headless client that implements the Webkit browser engine and has a JavaScript API. Phantomjs is used extensively for Web benchmarking and testing [49].

Figure 2 shows the median page load time of Mirage and Phantomjs for an emulated $10 \mathrm{Mbits} / \mathrm{s}$ access link with a last-mile latency of $40 \mathrm{~ms}$; the results also hold for lower latency (10 ms) links. Mirage always underestimates the actual page load time because it downloads fewer objects than a real browser would. Depending on the amount of dynamic content on the site, the difference may vary: in some cases, Mirage underestimates load times by up to $50 \%$; in others, its measured page load time is close to the load time that Phantomjs sees.

The implementation differences between Mirage and real browsers imply that the page load times that Mirage sees may not reflect the times that any real browser would see. Yet, page load times will always differ across different browsers, and we do not aim to estimate page load time from any particular browser. Our goal is to illustrate how components of network latency (e.g., DNS lookup time, TCP connect time) contribute to Web page load times. Mirage decomposes Web page load time into these components, which will be the same regardless of browser or any optimizations that a browser might perform. Mirage also allows us to evaluate how optimizations that a browser might perform can mitigate vari- 


\begin{tabular}{|c|c|c|c|c|c|c|c|c|c|c|c|c|}
\hline \multirow{3}{*}{ Target } & \multicolumn{3}{|c|}{ Objects } & \multicolumn{3}{|c|}{ Lookups } & \multicolumn{3}{|c|}{ Connections } & \multicolumn{3}{|c|}{ Size (KB) } \\
\hline & \multirow{2}{*}{$\begin{array}{r}\text { Sam- } \\
\text { Knows }\end{array}$} & \multicolumn{2}{|c|}{ BISmark } & \multirow{2}{*}{$\begin{array}{l}\text { Sam- } \\
\text { Knows }\end{array}$} & \multicolumn{2}{|c|}{ BISmark } & \multirow{2}{*}{$\begin{array}{r}\text { Sam- } \\
\text { Knows }\end{array}$} & \multicolumn{2}{|c|}{ BISmark } & \multirow{2}{*}{$\begin{array}{r}\text { Sam- } \\
\text { Knows }\end{array}$} & \multicolumn{2}{|c|}{ BISmark } \\
\hline & & US & non-US & & US & non-US & & US & non-US & & US & non-US \\
\hline edition.cnn.com & 26 & 25 & 26 & 4 & 4 & 4 & 12 & 12 & 12 & 1199 & 1022 & 1023 \\
\hline www. amazon.com & 24 & 31 & 32 & 4 & 4 & 4 & 21 & 24 & 23 & 589 & 840 & 851 \\
\hline www. ebay . com & 29 & 33 & 32 & 12 & 14 & 14 & 16 & 17 & 19 & 595 & 613 & 615 \\
\hline www. facebook.com & 8 & 8 & 7 & 2 & 2 & 2 & 7 & 8 & 7 & 437 & 389 & 289 \\
\hline www.google.com/mobile & 32 & 20 & 20 & 1 & 1 & 1 & 8 & 8 & 8 & 1398 & 289 & 291 \\
\hline WwW.msn.com & 24 & 24 & 54 & 8 & 8 & 8 & 14 & 14 & 16 & 377 & 348 & 641 \\
\hline wWW.wikipedia.org & 16 & 15 & 16 & 1 & 1 & 1 & 16 & 15 & 15 & 56 & 56 & 56 \\
\hline www $\cdot$ yahoo.com & 74 & 69 & 66 & 7 & 7 & 8 & 32 & 32 & 29 & 927 & 887 & 818 \\
\hline www youtube. com & 8 & 7 & 8 & 2 & 2 & 2 & 9 & 8 & 8 & 488 & 423 & 414 \\
\hline
\end{tabular}

Table 2: Properties of the Web sites in our data set. The values represent the average of the parameters over all transactions. Objects denotes the number of objects that must be downloaded; lookups are the number of DNS lookups required; and connections are the number of unique TCP connections the client set up to download all objects in the page. The number of connections depends on whether the server supports persistent connections, whether the objects are located on one or more domains, and the order in which objects are retrieved. Size denotes the number of bytes for all of the objects for a site.

\begin{tabular}{|l|r|r|r|}
\hline ISP & $\begin{array}{r}\text { Number of } \\
\text { homes }\end{array}$ & $\begin{array}{r}\text { Avg. last-mile } \\
\text { latency (ms) }\end{array}$ & $\begin{array}{r}\text { Avg. downstream } \\
\text { tput (Mbits/s) }\end{array}$ \\
\hline AT\&T & 718 & 18 & 8.7 \\
Cablevision & 209 & 6 & 34.1 \\
CenturyLink & 248 & 23 & 5.8 \\
Charter & 579 & 7 & 27.4 \\
Comcast & 932 & 8 & 22.8 \\
Cox & 618 & 7 & 21.2 \\
Mediacom & 242 & 14 & 18.7 \\
TimeWarner & 952 & 8 & 16.0 \\
Qwest & 299 & 30 & 10.0 \\
Verizon & 608 & 5 & 38.0 \\
Windstream & 251 & 20 & 5.9 \\
\hline
\end{tabular}

Table 3: The SamKnows deployment in the US.

Page load times for users outside the US are significantly higher for many sites; these higher times result mostly because of higher latencies to these sites.

Page load times stop improving beyond a downstream throughput of about $16 \mathrm{Mbits} / \mathrm{s}$. For higher throughputs, latency is the performance bottleneck.

The time to first byte can exceed the object load time in the cases of many small objects and for some pages can be as much as $21 \%$ of the page load time. The last-mile latency can be up to $23 \%$ of the time to first byte.

Table 5: Highlights of Section 5 results.

\begin{tabular}{|l|r|r|r|}
\hline Location & $\begin{array}{r}\text { \# of } \\
\text { homes }\end{array}$ & $\begin{array}{r}\text { Avg. last-mile } \\
\text { latency (ms) }\end{array}$ & $\begin{array}{r}\text { Avg. downstream } \\
\text { tput (Mbits/s) }\end{array}$ \\
\hline US & 43 & 12 & 24.2 \\
Europe & 3 & 24 & 8.3 \\
N. Amer. (non-U.S.) & 3 & 15 & 5.5 \\
E. Asia/Australia & 4 & 3 & 46.5 \\
Southeast Asia & 8 & 12 & 5.7 \\
\hline
\end{tabular}

Table 4: The BISmark deployment across the world.

ous network bottlenecks under different network conditions. When we evaluate the effects of different optimizations in Section 7, we again "close the loop" by showing that the benefits as predicted by Mirage are realized, even from a browser that is already performing its own optimizations.

\subsection{Deployment}

We use data from the FCC/SamKnows deployment, spanning 5,556 homes in the US; and the BISmark deployment, spanning 61 homes across the world. For both deployments, we use Mirage to characterize nine sites as chosen by SamKnows/FCC. Because it is difficult to measure a large number of Web sites from home routers and constrained access links, we focus on nine of the most popular sites around the world. Table 2 shows the sites that we measure and their properties. All of the measurements from both deployments are public. In some cases, the properties of the sites are different across deployments. Because both deployments use the same tool, these differences may result from either the vantage point or the time that the measurements were conducted. Because we do
§5.1, Fig. 3

$\S 5.2$, Fig. $5 \mathrm{a}$

§5.3, Fig. 6b . 


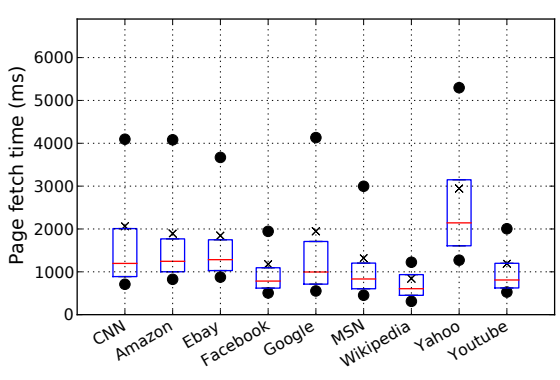

(a) SamKnows

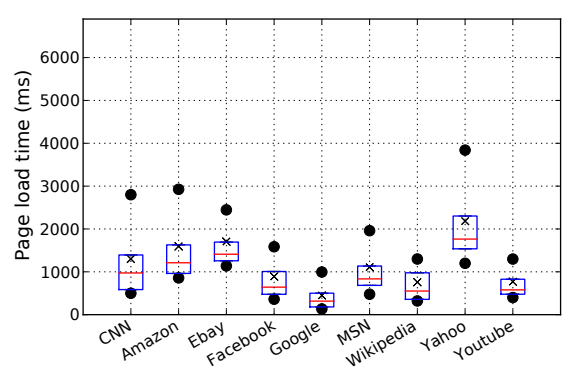

(b) BISmark-US

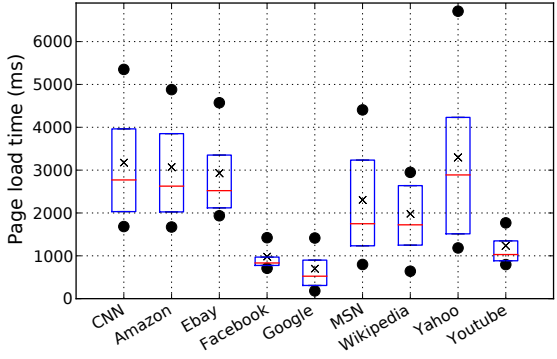

(c) BISmark-nonUS

Figure 3: Page load times for popular sites. The lower edge of each box indicates the $25^{\text {th }}$ percentile of the distribution of page load times for each site, the upper edge is the $75^{\text {th }}$ percentile, the middle line is the median, the cross represents the average, and the dots the $10^{\text {th }}$ and $90^{\text {th }}$ percentile page load times.

ceeds about $16 \mathrm{Mbits} / \mathrm{s}$. Last-mile latency (i.e., the latency between the home network and the first hop in the access ISP) is a significant overall contributor to both DNS lookup times and the time to first byte. Therefore, even when Web caches are deployed at the edge of access ISPs, optimizations in the home network that reduce the effects of last-mile latency can still offer significant performance improvements in page load time.

\subsection{Page Load Times of Popular Web Sites}

We study the page load times to nine popular Web sites. Figure 3 a shows the load times for access links in the SamKnows deployment while Figure $3 \mathrm{~b}$ shows load times for links in the BISmark deployment (both in the US). Figure 3c shows page load times for links in the BISmark deployment outside of the US. As expected, page load time varies both by site and the location of the access network. Some variability results from differences in page size and design (see Table 2); the largest four sites (CNN, Yahoo, Amazon, and Ebay) also have the largest load times (e.g., the median for CNN in the US is more than one second).

Figure $3 \mathrm{c}$ shows that access links outside of the US typically experience higher page load times for a given site than links in the US. The median and variance is higher for all sites we measure from outside the US, as well. A few sites have different sizes depending on the from which the page is requested, but most performance differences result from the fact that content is farther away from clients that are outside of the US. Figure 4 illustrates this phenomenon; the figure shows that the average time to first byte is in general higher in most regions outside the US. Our measurements also indicate that the global deployment of content distribution networks is somewhat spotty for certain sites. Sites with more expansive CDNs (e.g., Google, YouTube) have low median and maximum page load times, whereas other sites have more variable performance, both in the US and abroad. Even Google has relatively poor performance from Southeast Asia; we discussed this phenomenon with network operators at Google, who confirmed that Google's CDN deployment is not extensive in that region.

\subsection{Effects of Downstream Throughput}

We study how page load time and its components vary with downstream throughput using measurements from the SamKnows deployment. We use the $95^{t h}$ percentile of the distribution of downstream throughput over the duration of the measurements for a given user to capture the capacity of each access link. We group access links according to downstream throughput into seven bins that reflect common ranges of Internet access plans in the dataset:
0-1 Mbits/s, 1-2 Mbits/s, 2-4 Mbits/s, 4-8 Mbits/s, 8-16 Mbits/s, 16-32 Mbits/s, and 32-64 Mbits/s. Figure 5a shows the median page load time for each category for five representative sites.

Median page load time decreases as downstream throughput increases, up to about $16 \mathrm{Mbits} / \mathrm{s}$. As downstream throughput increases further, page load times decrease only modestly. For example, the median time for $\mathrm{CNN}$ is 8.4 seconds for links with throughput $0-1 \mathrm{Mbits} / \mathrm{s}$ and 1.3 seconds when throughput is $8-16 \mathrm{Mbits} / \mathrm{s}$. Yet, when downstream throughput exceeds $32 \mathrm{Mbits} / \mathrm{s}$, the page load time is $790 \mathrm{~ms}$, only slightly better than for links with 8$16 \mathrm{Mbits} / \mathrm{s}$

We study how each component of page load time varies with access link throughput. Page load time is heavily influenced by the maximum DNS lookup time and the maximum time to download a single object (regardless of any caching, parallel lookups, or other optimizations), so we can interpret the maximum times for these values as a lower bound on page load time. Large objects also have a correspondingly lower TCP overhead. Figure $5 \mathrm{~b}$ shows how these values decrease as throughput increases; each point shows the median value for the group of hosts with the corresponding range of downstream throughput. As downstream throughput increases to 32-64 Mbits/sec, object load time decreases from 3.2 seconds to $530 \mathrm{~ms}$. In contrast, the time to first byte decreases as the throughput of the link increases from $0-1$ to $1-2 \mathrm{Mbits} / \mathrm{s}$, but does not improve further for higher values of throughput. DNS lookup time decreases from about $50 \mathrm{~ms}$ to about $15 \mathrm{~ms}$. In summary, as downstream throughput increases beyond $8-16 \mathrm{Mbits} / \mathrm{sec}$, time to first byte and DNS times become a larger component of page load time and depend more on latency than on throughput-even for large objects.

Page load times for clients outside of the US illustrate the effect of latency on page load time. For example, the average page load time for Facebook is 1.7 seconds in southeast Asia, $990 \mathrm{~ms}$ in east Asia and Australia, and $924 \mathrm{~ms}$ in Europe. Table 4 shows that clients in east Asia have higher average throughput than Europe, but do not necessarily see a corresponding improvement in page load times because latency bottlenecks negate the effects of higher throughput.

\subsection{Effects of Last-Mile Latency}

Our previous study of access links in the US observed that lastmile latency contributes significantly to end-to-end latencies [55]. To study the effect of last-mile latency on page load times, we group access links into $10 \mathrm{~ms}$ bins, according to the 10th percentile last-mile latency. Figure 6 a shows the median page load time for 


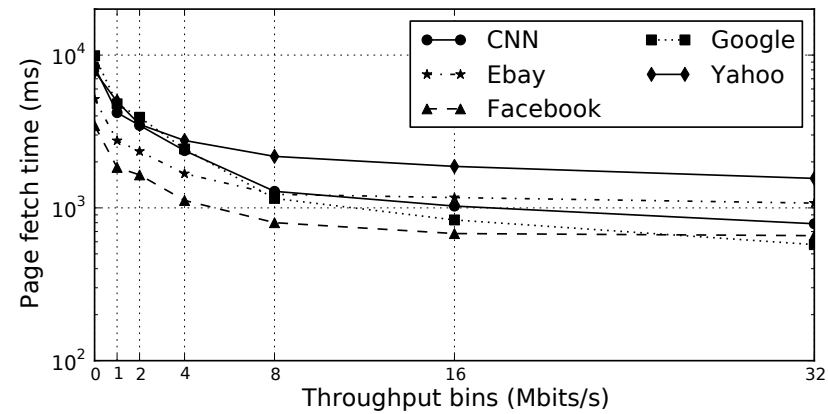

(a) Page load times

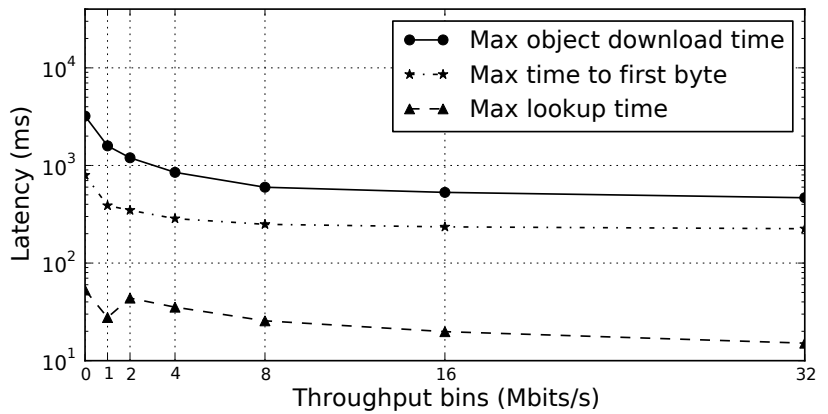

(b) Components of page load time

Figure 5: Page load times decrease with downstream throughput, but only up to 8-16 Mbits/s. X-axis labels denote the start of each throughput bin (e.g., "O" is the set of users with downstream throughput up to 1 Mbits/s.) (SamKnows)

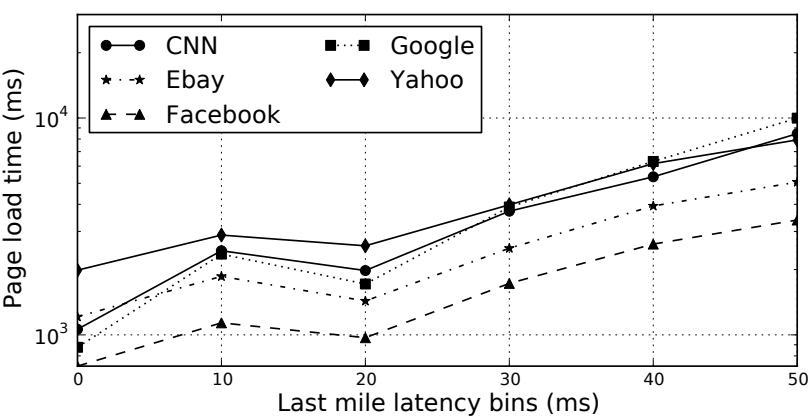

(a) Page load times

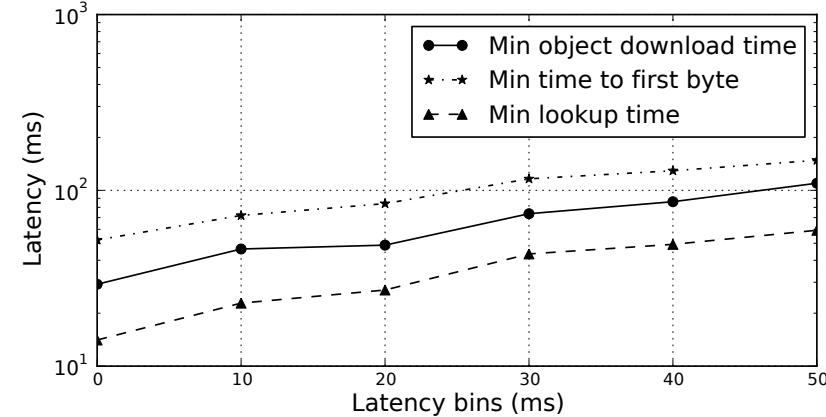

(b) Components of page load time

Figure 6: Page load times increase with last-mile latency. X-axis labels denote the start of each latency bin. (SamKnows)

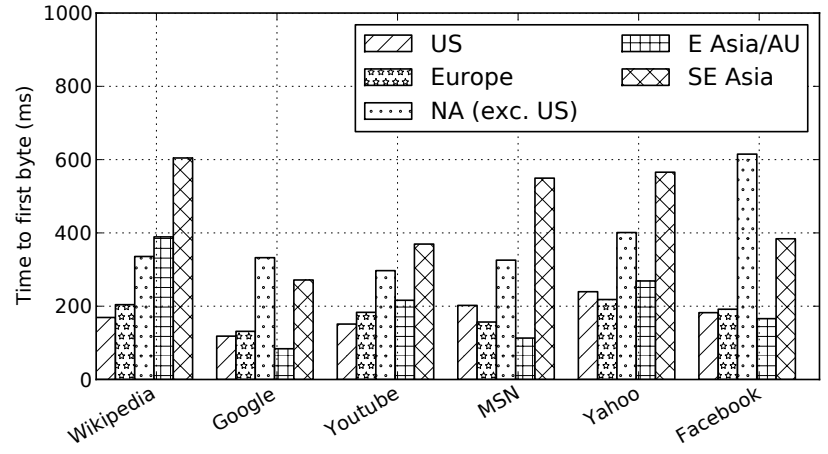

Figure 4: Average time to first byte to six representative sites from BISmark clients broken down by location.

each group for five representative sites. In general last-mile latency has a multiplicative effect on page load time, which is intuitive because it affects all packets in the transaction. The increase we see is not monotonic because other factors such as downstream throughput also affect page load time and some groups have more links than others: $75 \%$ of links have less than $10 \mathrm{~ms}$ last-mile latency. Even accounting for differences in throughput, an increase in lastmile latency of only $10 \mathrm{~ms}$ can result in a few hundred milliseconds increase in page load time. Increasing downstream throughput be- yond $16 \mathrm{Mbits} / \mathrm{s}$ yields only marginal improvements, but decreasing last-mile latency can have a larger impact on page load time.

To understand the overhead of TCP on small objects, we look at the minimum object download time and compare it to the minimum time to first byte and DNS lookup time. Figure $6 \mathrm{~b}$ shows the median of each of these values for each group of links. For smaller objects, the time to establish the TCP connection to the server is often greater than the time to actually transfer the object. Although the effect is magnified in smaller objects, we saw that it exists across all objects; in fact, we found that the average time to first byte ranges from $6.1 \%$ (for Yahoo) to $23 \%$ (for Wikipedia) of the total page load time. We also observed that last-mile latency can be as much as $23 \%$ of the time to first byte.

\subsection{Conclusion: Optimize Latency}

The results in this section suggest that for the increasing number of homes that have downstream throughput above $16 \mathrm{Mbits} / \mathrm{s}$, the most effective way to reduce Web page load time is to focus on reducing latency as much as possible.

Improving Object Fetch Time. Because most pages have many objects, the time to download all of them can dominate other factors when downstream throughput is small. Links with less than $16 \mathrm{Mbits} / \mathrm{s}$ will thus benefit the most from content caching. For links with higher downstream throughput, however, reducing object download time is less important than reducing latency. Thus, intuition suggests that faster access links can realize significant improvements in Web page load time simply by caching DNS records 
and TCP connections, without even having to cache any content. Our evaluation in Section 6 confirms this intuition.

Improving DNS Lookup Time. Figure $6 \mathrm{~b}$ shows that minimum lookup time increases as last-mile latency increases. Minimum lookup times are about $15 \mathrm{~ms}$, and they increase as the last-mile latency increases. The only approach to eliminate the last-mile latency is to cache DNS records inside the home itself. Even when the resolver is well-placed in the access ISP network, DNS lookup latencies are bound by last-mile latency (previous work confirms this observation [3]).

Improving Time to First Byte. Optimizations in the home network cannot improve server processing time, but they can improve TCP connection setup time. The connection setup time depends on the round-trip latency to the server. Web service providers use content distribution networks to place servers as close to users as possible. Figure 4 shows that servers are in general closer to homes in the US, but even users in the US can experience slowdowns in TCP connection setup time due to the last-mile latency. Clientside optimizations such as connection caching [31] can reduce this latency by maintaining TCP connections to popular sites, thereby reducing overhead for new connections.

\section{THE CASE FOR HOME CACHING}

We use Mirage to evaluate the benefits of DNS caching, TCP connection caching, and content caching in a home network. Although the optimizations that we evaluate are well known, the placement of these optimizations in the home router is new. Our analysis in this section offers two important contributions: (1) It is the first study to quantify the benefits of deploying them in the home network, where many users now access Web content; (2) To our knowledge, it is also the first study to quantify both the relative benefits to DNS caching, TCP connection caching, and content caching across a large number of popular sites and the holistic benefits of performing all three optimizations together.

Table 6 summarizes the main results in this section. Perhaps the most important takeaway from this section is that optimizations that reduce latency can significantly reduce page load time, even if content caching is not used. This result offers good news, since so much Web page content is dynamic, and since caching a large amount of content on end-hosts or inside home networks may prove infeasible. This finding also emphasizes the importance of placing optimizations inside the home network, since even the last-mile access link can introduce additional latency on the order of tens of milliseconds [55]. Similar optimizations already exist in the browser; we explain how a home cache provides additional benefits beyond browser-based optimizations in Section 7 .

\subsection{Experiment Setup}

We develop a controlled experiment to investigate how deploying three different caching optimizations in the home-DNS caching, TCP connection caching, and content caching - contribute to reducing page load time. We use the BISmark deployment described in Section 4 for our measurements. Mirage runs on the BISmark router; it uses a locally running DNS resolver and an HTTP proxy. dnsmasq is a lightweight caching DNS resolver [27] that caches up to 150 domains and honors the TTL values of the lookups. To evaluate TCP connection and content caching, we use polipo, a HTTP proxy that splits the TCP connection by opening a connection to the requested domain on behalf of the client and communicates with the client over a separate connection and
Performing DNS caching inside the home can improve maximum lookup times by about $15-50$ milliseconds, compared to simply relying on the ISP's DNS resolver.

Connection caching reduces median page load time by 100-750 milliseconds depending on the Web site.

Placing a content proxy inside the home versus in a nearby network improves median page load times between 150 and 600 milliseconds, depending on the site.

Applying all three optimizations together can reduce Web page load times by up to $60 \%$ at the router.

Table 6: Highlights of Section 6 results.

\begin{tabular}{|c|c|c|c|c|}
\hline Measurement & Proxy Location & DNS & Conn. & Content \\
\hline \multicolumn{5}{|c|}{ Baseline Measurements } \\
\hline No Proxy, ISP DNS & - & & & \\
\hline Cold Home Proxy & - & - & & \\
\hline ISP Proxy & Network & $\bullet$ & - & - \\
\hline \multicolumn{5}{|c|}{ Optimizations } \\
\hline Home DNS & Home & $\bullet$ & & \\
\hline Home Conn. Caching & Home & $\bullet$ & - & \\
\hline Home Proxy & Home & $\bullet$ & $\bullet$ & • \\
\hline
\end{tabular}

Table 7: The measurements we perform to evaluate the benefits of DNS, connection, and content caching in the home.

reuses TCP connections where possible. We run polipo with a 4 MByte cache in RAM.

Measuring Baseline Performance. Table 7 illustrates how we measure baseline performance and compare it with performance for each optimization. To measure the three baseline performance measurements, the client first performs the following three sets of requests:

1. ISP DNS Cache ("No Proxy, ISP DNS"). The client clears the local DNS cache and fetches the page directly from the server. This measures the baseline performance of fetching the Web page. The DNS lookups required for this measurement may reflect caching in the ISP's DNS resolver.

2. Empty Caching Proxy in the Home ("Cold Home Proxy"). The client fetches the same page by directing the request through a fresh polipo instance running in the router. Because polipo's cache is empty at this point, this measurement reflects the performance of a "cold" proxy. This step takes advantage of any DNS caching that dnsmasq performs in the previous step.

3. Shared ISP Caching Proxy ("ISP Proxy"). We cannot control a Web cache in a real ISP's network, so we approximate the behavior of an ISP cache by deploying a caching proxy in our university network. To measure the benefits of performing DNS, connection, and content caching at a shared proxy (the most common setup for content caches), the client first fetches the page through a polipo proxy running on a university server to warm the cache. It then immediately repeats the step. We perform the measurements from eleven BISmark routers that are less than $35 \mathrm{~ms}$ away from the proxy, so that it emulates nearby ISP caches.

Quantifying the Benefits of Caching in Home Networks. After collecting the baseline measurements, the client then performs 
three additional requests to measure the relative benefit of performing different optimizations in the home.

4. Home DNS caching only ("Home DNS"). The client fetches the same page directly from the servers. This measures the benefits of DNS caching in the home (since dnsmasq caches the DNS responses from earlier measurements).

5. Home proxy with DNS caching, persistent connections, and content caching ("Home Proxy") The client fetches the page through the local polipo again; this measurement takes advantage of DNS, content and connection caching, since the proxy would have cached any cacheable objects and reused TCP connections where possible from the requests in the "Cold Home Proxy" experiment.

6. Home proxy with DNS caching and persistent connections only ("Home Connection Caching") The client clears the polipo cache on the home router - this gets rid of the content in the cache, but keeps the TCP connections from the previous step alive. It then fetches the page through the proxy again. All content is retrieved again from the origin service, but the TCP connections from the previous step are reused.

These experiments allow us to isolate the effects of (1) the relative benefits of performing DNS, connection, and content caching inside the home network and (2) placing a cache inside the home versus elsewhere (e.g., in the ISP).

\subsection{Effects of Home Caching on Latency}

Benefits of DNS caching in the home vs. in the ISP. To quantify the benefits of DNS caching in the home, we compare the maximum lookup time for the Home DNS Cache and No Proxy, ISP $D N S$ cases. Figure 7 shows the CDF of the improvement in the maximum lookup time for the page load. In the median case, DNS caching in the home reduces the maximum lookup time by 15$50 \mathrm{~ms}$, depending on the site. Certain clients outside the US can reduce their lookup time by several hundred milliseconds for certain sites like Ebay and CNN by caching DNS responses in the home. For a small fraction of cases, DNS caching in the home actually slightly impairs performance; in these cases, the detriment in small and may result from changes in network conditions between experiments (this effect occurs for some of our TCP connection caching and content caching experiments, too).

Benefits of TCP connection caching in the home. Figure 8 shows the additional improvement in page load time due to connection caching by measuring the difference between the load times for the Home Connection Caching and the Home DNS Cache measurements. The median improvement varies from 100-750 ms depending on the site. Ebay and Yahoo experience the most improvement in load times because both sites require many objects from many domains to render; connection caching can significantly reduce TCP overhead in such cases.

\subsection{Effects of Home Caching on Throughput}

Benefits of content caching vs. connection caching. Figure 9 shows the improvement in page load time due to content caching over connection caching. We compute the improvement by subtracting the page load time for the Home Proxy experiment from that for the Home Connection Caching experiment. Caching content inside the home can decrease median page load times in the US by 75-400 ms over connection caching, depending on the site. Obviously, sites with more cacheable content will benefit more. Our
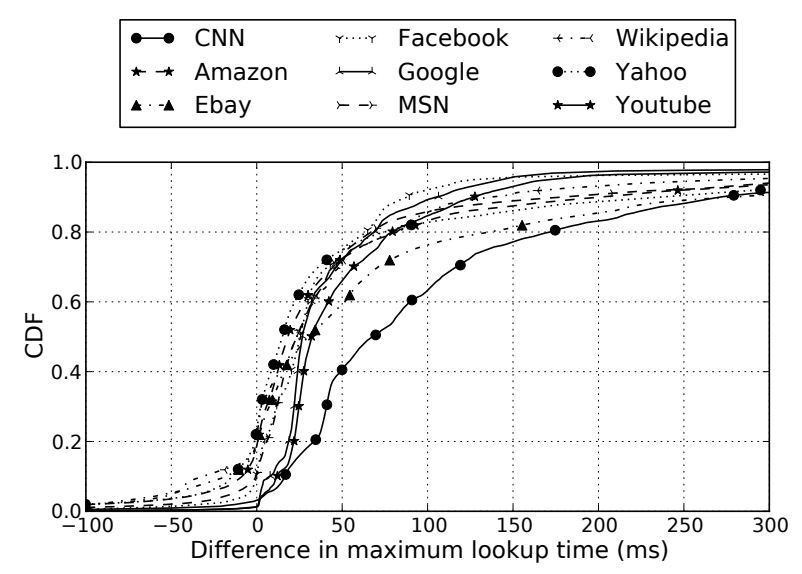

Figure 7: Caching DNS in the home can reduce the maximum DNS lookup time by 15-50 ms. (Home DNS Measurement vs. No Proxy, ISP DNS Measurement)

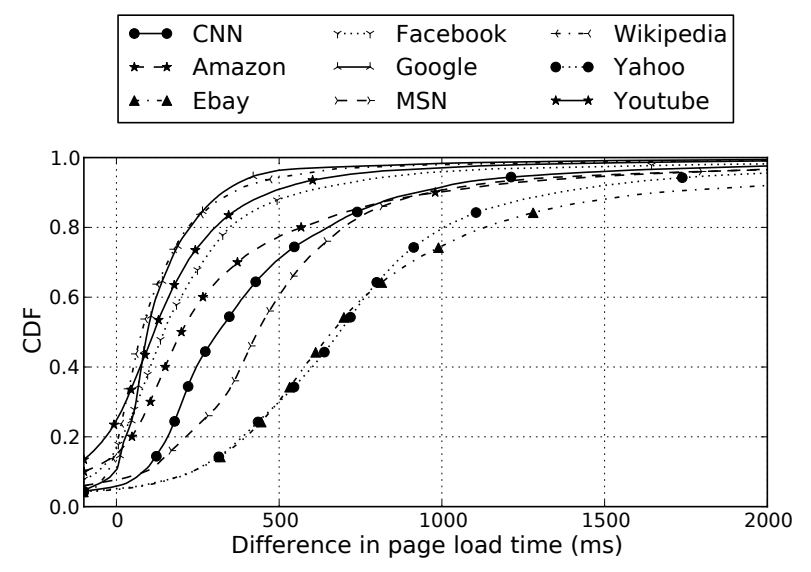

Figure 8: Connection caching in the home can reduce median page load times by 100-750 ms. (Home Connection Proxy vs. Home DNS Measurements)

analysis shows that this benefit is even more significant for clients outside the US; at least $20 \%$ of clients experienced an improvement of $500 \mathrm{~ms}$ or more for all sites.

Benefits of content caching in the home vs. in the ISP. We compare the Web page load time when using a remote HTTP proxy (the ISP Proxy measurement from Table 7) versus using a local HTTP proxy running on the router (the Home Proxy measurement). Figure 10 shows that a proxy in the home can offer a median improvement in page load time of $150-600 \mathrm{~ms}$, depending on the site. Yahoo and CNN experience the most benefits, likely because these pages are larger and have many objects (Table 2). A cache in the upstream ISP is still constrained by the access link's throughput and last-mile latency, while a local cache is not. For some sites, the remote proxy performs better than the home proxy about $20 \%$ of the time, perhaps because of varying access link characteristics across tests (due to cross traffic) or because the proxy is in a university network that potentially has better connectivity to these sites.

\subsection{Putting It Together}

We now quantify the collective benefit from performing all three optimizations. We use Mirage's No Proxy, ISP DNS measurement 


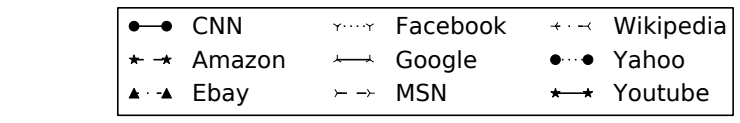

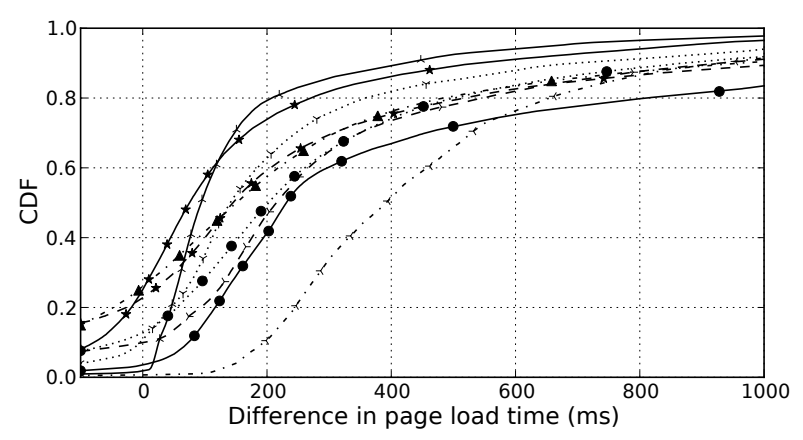

Figure 9: Content caching reduces the median page load time by 75-400 ms over connection caching alone. For sites with more cacheable content, the benefit is greater (Home Proxy vs. Home Connection Caching Measurements)

\begin{tabular}{|c|c|c|}
\hline $\begin{array}{l}\bullet \text { CNN } \\
\star \rightarrow \text { Amazon } \\
\star-\text { Ebay }\end{array}$ & $\begin{array}{l}\stackrel{r \cdots r}{ } \text { Facebook } \\
\longleftrightarrow \text { Google } \\
\succ \rightarrow \mathrm{MSN}\end{array}$ & $\begin{array}{l}+\cdots \text { Wikipedia } \\
\bullet \cdots \text { Yahoo } \\
\star \star \text { Youtube }\end{array}$ \\
\hline
\end{tabular}

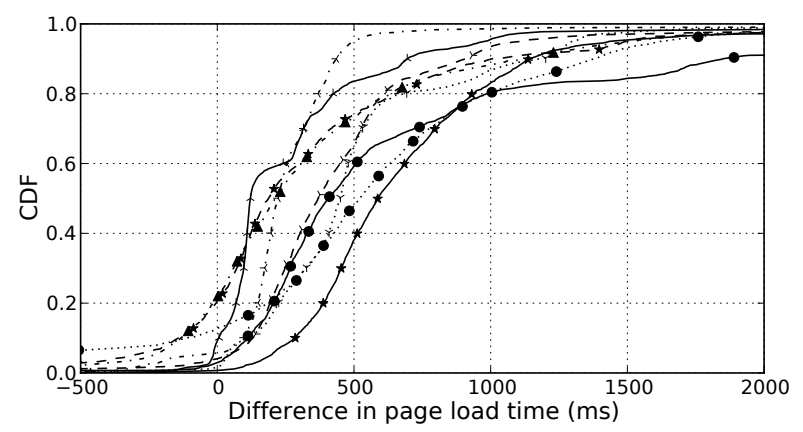

Figure 10: Running a proxy in the home improves median page load times by 150-600 ms versus running a proxy in the ISP. (Home Proxy vs. ISP Proxy Measurement)

as the baseline. We compute the relative improvement as $(b-v) / b$, where $b$ is the baseline load time and $v$ is the optimized load time.

Figure 11a shows the relative improvement of each optimization relative to the baseline of performing no optimizations in BISmarkUS. Applying all three optimizations improves page load time by as much as $60 \%$. Even without content caching, connection caching can yield up to a $35 \%$ improvement in load time, and DNS caching alone can improve page load time by as much as $10 \%$. Figure $11 \mathrm{~b}$ shows the relative improvement for clients outside the US. The improvement is slightly less than for the users within the US because the absolute page load times for users outside the US are already higher (Figure 3). The variance is also higher because of the wide range of operating conditions outside the US (Table 4 and Figure 4). The improvements we measure represent the best-case scenario because the quick succession of Web page retrievals will always induce a cache hit at the router. In the next section, we explore how to design a cache in the home that achieves high cache hit rates in practice.

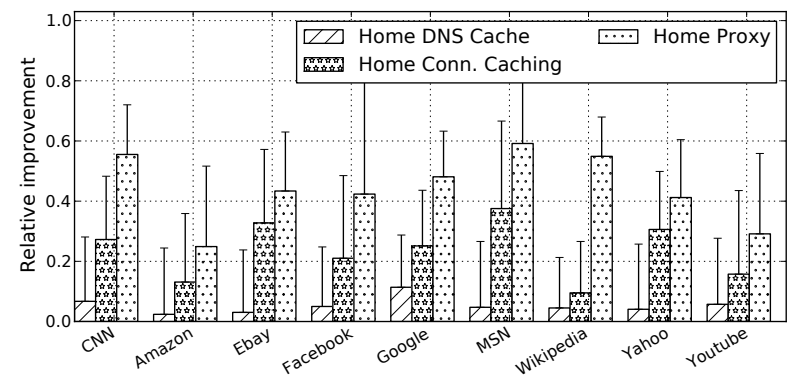

(a) BISmark-US

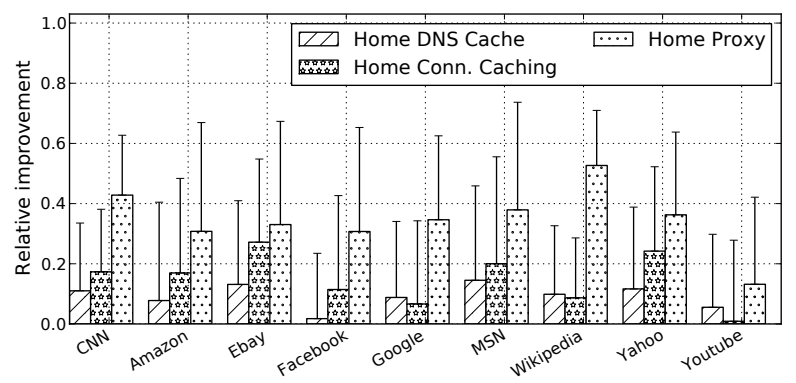

(b) BISmark-nonUS

Figure 11: Average relative improvement in page load times for various optimizations, as observed from the router. Error bars denote standard deviation.

\section{HOME CACHING IN PRACTICE}

The previous section demonstrated how home caching can improve page load times in the best-case scenario, but the results measured the benefits in isolation from other optimizations (e.g., browser caching) and also assumed that these cache hits could be realized in practice. Thus, our experiments from Section 6 raise two questions:

1. How can users inside a home network practically realize cache hits on a home cache? Prefetching DNS queries and maintaining open TCP connections to all sites visited by a user is not practical. To intelligently determine which DNS records and TCP connections to cache, we implemented a lightweight router-based popularity-based prefetching system that prefetches and caches DNS records and maintains active TCP connections to popular domains to help improve cache hit rates. We analyzed cache hit ratios resulting from this system with a trace-driven simulation using passive traces collected from twelve homes. (Section 7.1)

2. Are the performance benefits that home caching offers significant, in light of the fact that browsers already perform some amount of caching and prefetching? To answer this question, we retrieve Web pages from a laptop in a home network using Phantomjs under a variety of emulated access link characteristics with various caching optimizations enabled on the home router. (Section 7.2)

Our results indicate that popularity-based prefetching can complement existing browser optimizations. 


\subsection{Popularity-based Prefetching}

We design, implement, and evaluate a popularity-based caching and prefetching systemthat prefetches DNS records and keeps TCP connections to Web servers active based on the sites that users in the household visit most frequently. We develop a proof-of-concept OpenWrt module, which is publicly available [50]. The system consists of (dnsmasq) and (polipo), instrumented to track popular DNS lookups and HTTP domains respectively. The system tracks popularity and refreshes DNS lookups and maintains an active TCP connection to popular domains by using a simple caching mechanism. The system aims to maximize the hit rate of the DNS and TCP connection caches. The two parameters that affect the hit rate are (1) the number of domains to be tracked: the system actively prefetches DNS records and maintains active connections to these domains; the system maintains the two lists separately; and (2) timeout thresholds: the system tracks the time since a lookup or a TCP connection was requested to a domain and removes the domain from the popular list if this time exceeds a threshold. The system does not prefetch content but exploits any content caching that polipo performs by default.

Does popularity-based prefetching improve Web page load times in homes? To analyze whether popularity-based prefetching can improve performance, we perform a trace-based simulation using real browsing activity in home networks, with optimizations enabled and disabled. We capture Web browsing activity of users from routers deployed in twelve homes in the US for the period October 15-31, 2012 to evaluate the potential improvements from proactive DNS and connection caching in home networks. We capture DNS and HTTP traffic. We record the timestamp, hashed domain, and TTL of all resolved DNS responses. Each HTTP flow event has a timestamp of all connections, hashes of their corresponding IP addresses, flow duration, and TCP connection time. To preserve user privacy, we do not capture packet payloads, so we do not evaluate cache hit ratios for content.

Using these traces, we run a trace-driven simulation that maintains the DNS cache using the timestamp, hashed domain, and TTL of resolved DNS responses from the trace. When prefetching a domain, the simulation delays each incoming DNS response by a random interval between 50 and $200 \mathrm{~ms}$; the TTL value is set according to the value in the trace. Requests for domains waiting for a DNS response are considered cache misses. To characterize the benefit of TCP connection caching, the simulation maintains the TCP connection cache based on the timestamp, five-tuple flow identifier, and duration of both the entire TCP connection and its connection establishment of all port 80 requests from the passive traces. For each new request, the simulation compares it with the entries in the connection cache. In the event of a cache miss, or when a connection to an IP address is in use by another request, we establish a new connection with the flow and TCP connection establishment durations that correspond to the TCP connection in the trace. Upon a cache hit, the simulation denotes the cache entry to be in use for the duration of TCP connection minus the connection establishment time to that IP.

Baseline DNS cache hit rates without popularity-based prefetching vary from $11-50 \%$, depending on the home; the baseline cache hit ratio for TCP connection ranges from 1-8\% across homes. Setting a cache size of 20 and a timeout threshold of 120 seconds for both DNS and connection prefetching causes DNS hit ratios improve to $19-93 \%$, representing at least a $40 \%$ improvement over the baseline in every home. Connection cache hit ratios increase to $6-21 \%$ across homes. These results show that popularity-based

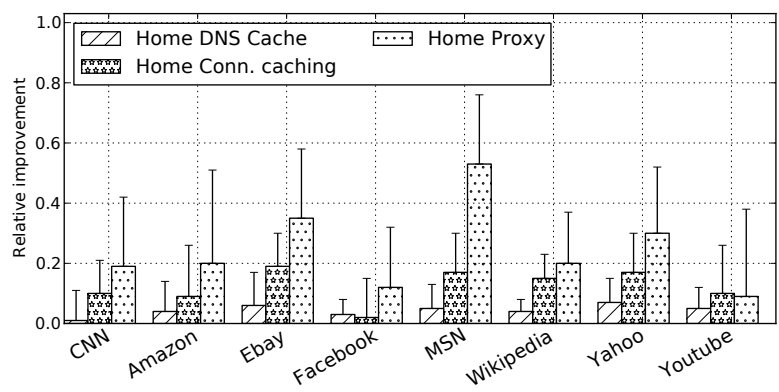

Figure 12: Average relative improvement in page load times for various optimizations, as observed from the browser. Error bars denote standard deviation.

prefetching for only a few sites and with a short timeout can significantly improve performance.

\subsection{Benefits of Home Caching from Browsers}

We evaluate the benefit of home caching as seen from a browser by analyzing the improvement in page load times from Phantomjs with various caching optimizations enabled. We measure page load times from a Web browser using Phantomjs running on a Linux laptop that is connected through a wired link to a Netgear home router; the router shapes the uplink to represent different home network throughputs and latencies. We use two settings for the downstream throughput of the access link ( $10 \mathrm{Mbits} / \mathrm{s}$ and $20 \mathrm{Mbits} / \mathrm{s}$ ) and three settings for the latency of the access link $(0 \mathrm{~ms}, 20 \mathrm{~ms}$, and $40 \mathrm{~ms})$. We download each of the sites 25 times for each setting. These parameters approximate common access-link throughputs and lastmile latencies. The router also runs popularity-based prefetching, discussed in Section 7.1.

Home caching complements browser optimizations. Figure 12 shows the relative improvement in Web page load times as a result of deploying various caching optimizations from a home router, as observed from the laptop running Phantomjs. Because we had problems with Phantomjs using polipo for Google, we omit results for it. DNS caching improves page load times by as much as 7\%; connection and DNS caching improve load times by about $20 \%$; all three optimizations together reduce load times by as much as $60 \%$, depending on the site. The benefits as measured using Phantomjs are lower, but they are comparable to what we observe at the router in Figure 11. Although the improvements in page load time that a browser realizes obviously depends on many factors, our results demonstrate that home caching complements existing optimizations that browsers already perform.

To further illustrate these benefits, we show the page load time for Ebay, for a single experiment. Figure 13 shows how DNS and TCP connection caching improves per-object downloads. It shows the time to download the first ten objects of the site using Phantomjs. We measure the total time for the first ten objects. The left plot shows the baseline case without optimization, and the second plot shows the benefits that the browser observes when the home router performs DNS and connection caching. We observe an $6.4 \%$ reduction in page load time for the home page alone when the home router performs both DNS and TCP caching. The improvements are relatively more significant for smaller objects. The numbers next to the objects show the percentage improvement in the baseline. All objects show some improvement; objects that are cacheable see more improvement. 

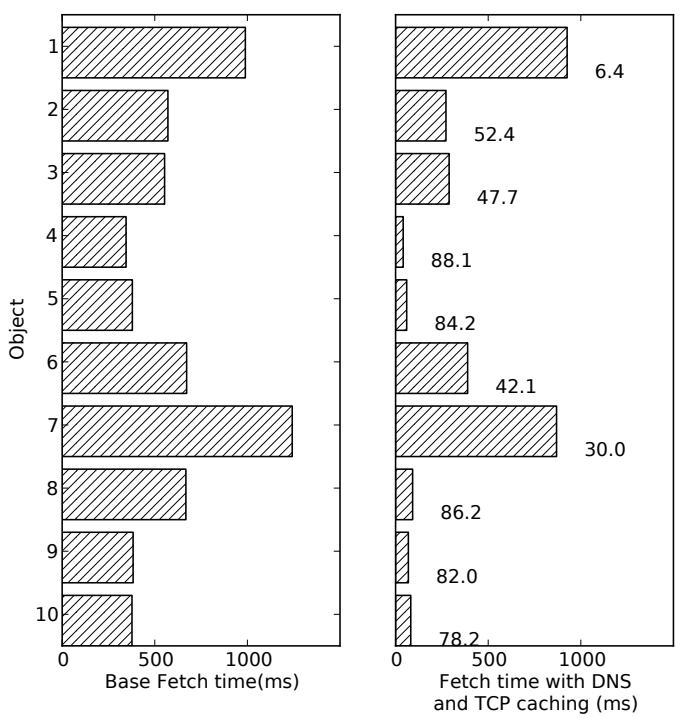

Figure 13: The time to load the first ten objects for Ebay using Phantomjs. We plot the total download time for the first ten objects. The first plot shows the breakdown for the base case; the second when there is DNS and TCP connection caching. The numbers in the second plot denote the percentage improvement over the base case.

\section{CONCLUSION}

We presented the first large-scale study of Web performance bottlenecks in broadband access networks. We first characterize performance to nine popular Web sites from 5,556 access networks using Mirage, a router-based Web measurement tool, and identify factors that create Web performance bottlenecks. Regardless of the optimizations that clients, servers, and browsers may perform, the access link is a fundamental limit to the efficacy of optimizations. As throughput increases, latency becomes more important, to the extent that even having caches on the edge of ISPs could prove to be insufficient. We show that Web page load times stop improving as throughput rates increase beyond $16 \mathrm{Mbits} / \mathrm{s}$. We also show that last-mile latency contributes to the time required for performing DNS lookups, completing TCP connection setup, and downloading Web objects and can be up to $23 \%$ of the time to first byte.

Moreover, we evaluate techniques to mitigate latency bottlenecks by implementing home caching, which performs DNS and TCP connection caching and prefetching inside the home network. We demonstrate that home caching can improve Web performance, even if the ISP and browser are already independently performing similar optimizations. TCP connection caching alone can improve Web page load times by up to $20 \%$, depending on the site. Performing all three optimizations together from the home router can reduce page load times by up to $53 \%$.

Although the optimizations we describe can be implemented anywhere in the home network, we believe that the home router is a reasonable location for caching and prefetching DNS records and TCP connections. In addition to further reducing page load times, even in the presence of similar optimizations that browsers already implement, it is worth noting that there are many Webbased applications that do not run from a standard browser. Any device in the home network that retrieves Web content can benefit from home caching. Indeed, as Singhal and Paoli state, "apps—not just browsers - should get faster too" [52]. Our publicly available OpenWrt module for popularity-based prefetching may serve as yet another important component for reducing page load times in home networks.

\section{Acknowledgments}

This research was funded in part by NSF award CNS-1059350, a Google Focused Research Award, and the European Community's Seventh Framework Programme (FP7/2007-2013) no. 258378 (FIGARO). We would like to thank the Federal Communications Commission and SamKnows for access to the data. We would also like to acknowledge BISmark volunteers worldwide for supporting the platform.

\section{REFERENCES}

[1] Tel-Aviv, Israel, Mar. 2000.

[2] Berlin, Germany, Nov. 2010.

[3] B. Ager, W. Mühlbauer, G. Smaragdakis, and S. Uhlig. Comparing dns resolvers in the wild. In Proceedings of the 10th annual conference on Internet measurement, IMC '10, pages 15-21, New York, NY, USA, 2010. ACM.

[4] B. Ager, F. Schneider, J. Kim, and A. Feldmann. Revisiting cacheability in times of user generated content. In INFOCOM IEEE Conference on Computer Communications Workshops, 2010, pages 1-6. IEEE, 2010.

[5] A. Akella, B. Maggs, S. Seshan, and A. Shaikh. On the performance benefits of multihoming route control. IEEE/ACM Transactions on Networking, 16(1), Feb. 2008.

[6] A. Akella, B. Maggs, S. Seshan, A. Shaikh, and R. Sitaraman. A measurement-based analysis of multihoming. In Proc. ACM SIGCOMM, Karlsruhe, Germany, Aug. 2003.

[7] A. Akella, J. Pang, B. Maggs, S. Seshan, and A. Shaikh. A comparison of overlay routing and multihoming route control. In Proc. ACM SIGCOMM, Portland, OR, Aug. 2004.

[8] A. Akella, S. Seshan, and A. Shaikh. Multihoming performance benefits: An experimental evaluation of practical enterprise strategies. In Proc. USENIX Annual Technical Conference, Boston, MA, June 2004.

[9] M. Al-Fares, K. Elmeleegy, B. Reed, and I. Gashinsky. Overclocking the Yahoo! CDN for faster Web page loads. In Proceedings of Internet Measurement Conference, 2011.

[10] E. Altman, K. Avrachenkov, and C. Barakat. A stochastic model of tcp/ip with stationary random losses. In ACM SIGCOMM, 2000.

[11] M. Arlitt, B. Krishnamurthy, and J. Mogul. Predicting Short-transfer Latency from TCP arcana: a Trace-based Validation. In Proc. ACM SIGCOMM Internet Measurement Conference, New Orleans, LA, Oct. 2005.

[12] P. Barford and M. Crovella. Critical path analysis of tcp transactions In IEEE/ACM Transactions on Networking, 2000.

[13] M. Belshe. A Client-Side Argument for Changing TCP Slow Start. http://goo.gl/UDKXz.

[14] M. Belshe. More Bandwidth Doesn't Matter (much). http://goo.gl/OIv47.

[15] BISmark Web Performance data. http: //dl.dropbox.com/ u/13045329/bismark_webperf_data.tar.gz.

[16] J. Brutlag. Speed matters for Google Web search. http://services.google.com/fh/files/blogs/ google_delayexp.pdf, June 2009.

[17] M. Butkiewicz, H. Madhyastha, and V. Sekar. Understanding website complexity: Measurements, metrics, and implications. In Proc. Internet Measurement Conference [2].

[18] R. Caceres, F. Douglis, A. Feldmann, G. Glass, and M. Rabinovich. Web proxy caching: The devil is in the details. June 1998.

[19] J. Cao, W. S. Cleveland, Y. Gao, K. Jeffay, F. D. Smith, and $\mathrm{M}$. Weigle. Stochastic models for generating synthetic http source traffic. In IN PROCEEDINGS OF IEEE INFOCOM, 2004. 
[20] N. Cardwell, S. Savage, and T. Anderson. Modeling tcp latency. In Proc. IEEE INFOCOM [1]

[21] Y. Cheng and Others. TCP Fast Open. IETF, Sept. 2011. http://www.ietf.org/id/ draft-cheng-tcpm-fastopen-00.txt.

[22] J. Chu and Others. Increasing TCP's Initial Window. IETF, Oct. 2011. http://tools.ietf.org/html/ draft-ietf-tcpm-initcwnd-01.

[23] E. Cohen and H. Kaplan. Prefetching the means for document transfer: A new approach for reducing Web latency. In Proc. IEEE INFOCOM [1], pages 854-863.

[24] E. Cohen and H. Kaplan. Proactive caching of DNS records: Addressing a performance bottleneck. In Symposium on Applications and the Internet (SAINT), pages 85-94, 2001.

[25] Compuware. http: //www. compuware.com/en_us/ application-performance-management/products/ application-aware-network-monitoring/ web-services / overview.html.

[26] DNS Prefetching (or Pre-Resolving). http: //blog.chromium.org/2008/09/ dns-prefetching-or-pre-resolving.html.

[27] Dnsmasq. http://thekelleys.org.uk/dnsmasq/doc.html.

[28] N. Dukkipati, T. Refice, Y. Cheng, J. Chu, T. Herbert, A. Agarwal, A. Jain, and N. Sutin. An argument for increasing tcp's initial congestion window. SIGCOMM Comput. Commun. Rev., 40:26-33, June 2010.

[29] J. Erman, A. Gerber, M. Hajiaghayi, D. Pei, and O. Spatscheck. Network-aware forward caching. In Proceedings of the 18th international conference on World wide web, 2009.

[30] FCC Measuring Broadband America Report. http: / / www . fcC. gov/measuring-broadband-america/2012/july, July 2012.

[31] A. Feldmann, R. Caceres, F. Douglis, G. Glass, and M. Rabinovich. Performance of web proxy caching in heterogeneous bandwidth environments. In Proc. IEEE INFOCOM, New York, NY, Mar. 1999.

[32] S. Gribble and E. Brewer. System Design Issues for Internet Middleware Services: Deductions from a Large Client Trace. In Proc. 1st USENIX Symposium on Internet Technologies and Systems (USITS), Monterey, CA, Dec. 1997.

[33] S. Ihm and V. Pai. Towards understanding modern web traffic. In Proc. Internet Measurement Conference [2].

[34] A. jan Su, D. R. Choffnes, A. Kuzmanovic, and F. E. Bustamante. Drafting behind akamai (travelocity-based detouring. In Proc. ACM SIGCOMM, Pisa, Italy, Aug. 2006.

[35] J. Jung, A. W. Berger, and H. Balakrishnan. Modeling TTL-based Internet Caches. In IEEE Infocom 2003, San Francisco, CA, April 2003.

[36] J. Jung, E. Sit, H. Balakrishnan, and R. Morris. DNS Performance and the Effectiveness of Caching. In Proc. ACM SIGCOMM Internet Measurement Workshop, San Fransisco, CA, Nov. 2001.

[37] Keynote. http://www. keynote.com/products/web_ performance/web-performance-testing.html.

[38] B. Krishnamurthy and C. Wills. Analyzing factors that influence end-to-end Web performance. In Proc. Twelfth International World Wide Web Conference, Amsterdam, The Netherlands, May 2000.

[39] R. Krishnan, H. V. Madhyastha, S. Jain, S. Srinivasan, A. Krishnamurthy, T. Anderson, and J. Gao. Moving beyond end-to-end path information to optimize CDN performance. In Proc. Internet Measurement Conference, 2009.

[40] Z. Li, M. Zhang, Z. Zhu, Y. Chen, A. Greenberg, and Y.-M. Wang. Webprophet: Automating performance prediction for web services. In Proc. 7th USENIX NSDI, San Jose, CA, Apr. 2010.
[41] Link Prefetching FAQ. https://developer.mozilla.org/ En/Link_prefetching_FAQ.

[42] S. Lohr. For Impatient Web Users, an Eye Blink Is Just Too Long to Wait.

http: / /www. nytimes.com/2012/03/01/technology/ impatient-web-users-flee-slow-loading-sites. html, Mar. 2012.

[43] J. C. Mogul, Y. M. Chan, and T. Kelly. Design, implementation, and evaluation of duplicate transfer detection in HTTP. In Proc. First Symposium on Networked Systems Design and Implementation (NSDI), San Francisco, CA, Mar. 2004

[44] H. Nielsen, J. Gettys, A. Baird-Smith, E. Prud'hommeaux, H. W. Lie, and C. Lilley. Network performance effects of http/1.1, css1, and png. In Proc. ACM SIGCOMM, Cannes, France, Sept. 1997.

[45] OECD. OECD Communications Outlook. OECD Publishing, July 2011.

[46] J. Padhye, V. Firoiu, D. Towsley, and J. Kurose. Modeling TCP Throughput: A Simple Model and its Empirical Validation. In Proc. ACM SIGCOMM, pages 303-323, Vancouver, British Columbia, Canada, Sept. 1998.

[47] V. Padmanabhan and J. Mogul. Using predictive prefetching to improve world wide web latency. ACM SIGCOMM Computer Communication Review, 26(3):22-36, 1996.

[48] Phantomjs. http://phantomjs.org/.

[49] Phantomjs Users. https://github.com/ariya/phantomjs/wiki/Users.

[50] OpenWRT Module for Popularity-based Prefetching. http://dl.dropbox.com/u/13045329/popularity_ prefetch.tgz.

[51] Project bismark. http: //projectbismark. net.

[52] S. Singhal and J. Paoli. Speed and Mobility: An Approach for HTTP 2.0 to Make Mobile Apps and the Web Faster, Mar. 2012. http: //goo.gl/1uWCl.

[53] S. Souders. Velocity and the bottom line. http://radar.oreilly.com/2009/07/ velocity-making-your-site-fast.html, July 2009

[54] SPDY: An experimental protocol for a faster web. http: //www. chromium.org/spdy/spdy-whitepaper.

[55] S. Sundaresan, W. de Donato, N. Feamster, R. Teixeira, S. Crawford and A. Pescapè. Broadband internet performance: A view from the gateway. In Proc. ACM SIGCOMM, Toronto, Ontario, Aug. 2011.

[56] S. Sundaresan, N. Magharei, N. Feamster, and R. Teixeira. Accelerating last-mile web performance with popularity-based prefetching. In Proceedings of ACM SIGCOMM 2012 (Poster), SIGCOMM '12. ACM, 2012.

[57] S. Sundaresan, N. Magharei, N. Feamster, R. Teixeira, and $\mathrm{S}$. Crawford. Web performance bottlenecks in broadband access networks. In Proceedings of ACM SIGMETRICS 2013 (Poster Paper), SIGMETRICS '13. ACM, 2013.

[58] X. S. Wang, A. Balasubramanian, A. Krishnamurthy, and D. Wetherall. Demystifying page load performance with wprof. Apr 2013.

[59] A. Wolman, G. M. Voelker, N. Sharma, N. Cardwell, A. Karlin, and H. M. Levy. On the scale and performance of cooperative web proxy caching. In Proc. 17th ACM Symposium on Operating Systems Principles (SOSP), Kiawah Island, SC, Dec. 1999.

[60] W. Zhou, Q. Li, M. Caesar, and P. Godfrey. Asap: A low-latency transport layer. In Proceedings of the Seventh COnference on emerging Networking EXperiments and Technologies, page 20 ACM, 2011. 